\title{
Identification of salsolinol in the mediobasal hypothalamus of lactating ewes and its relation to suckling-induced prolactin and $\mathrm{GH}$ release
}

\author{
Tomasz Misztal ${ }^{1}$, Konrad Górski ${ }^{1}$, Dorota Tomaszewska-Zaremba ${ }^{2}$, Edyta Molik ${ }^{3}$ \\ and Katarzyna Romanowicz ${ }^{1}$ \\ Departments of ${ }^{1}$ Endocrinology and ${ }^{2}$ Neuroendocrinology, The Kielanowski Institute of Animal Physiology and Nutrition, Polish Academy of Sciences, 05-110 \\ Jablonna $\mathrm{n} /$ Warsaw, Poland \\ ${ }^{3}$ Department of Sheep and Goat Breeding, Agricultural University in Cracow, Mickiewicza 24/28 Street, 30-059 Cracow, Poland \\ (Correspondence should be addressed to T Misztal; Email: t.misztal@ifzz.pan.pl)
}

\begin{abstract}
The push-pull perfusions of the infundibular nucleus-median eminence (IN/ME) were made in lactating ewes $(n=7)$ twice, to identify dopamine (DA)-derived salsolinol and the changes in its extracellular concentration in response to suckling. The perfusate collecting period in every ewe consisted of control non-suckling period, 1000-1230 h (five perfusates), and suckling period, 1230-1500 h (next five perfusates). Simultaneously, blood samples were collected from 1000 to $1500 \mathrm{~h}$ at $10-\mathrm{min}$ intervals. The perfusate concentrations of salsolinol and DA were measured by HPLC, and plasma prolactin and $\mathrm{GH}$ concentrations were assayed by the RIA. Mean concentrations of salsolinol in perfusates collected from the anterior and posterior parts of the IN/ME (according to post-mortem localization of a perfusion site) increased significantly $(P<0 \cdot 05$ and $P<0 \cdot 001$ respectively)
\end{abstract}

during the suckling period, when compared with those noted during the non-suckling period. While no DA was found in the anterior part, only vestigial amounts of DA were found in a few perfusates collected from the posterior part. Salsolinol was not detected in the IN/ME of ewes 10 weeks after weaning (seasonal anoestrus). Mean plasma prolactin and $\mathrm{GH}$ concentrations during suckling were significantly $(P<0 \cdot 001)$ higher than those noted during the non-suckling period. In conclusion, our current study reveals that salsolinol is present in the IN/ME of lactating ewes and that its extracellular concentration increases during suckling. Moreover, it supports the role of salsolinol as a neurotransmitter involved in the regulatory process of prolactin secretion at least during lactation.

Journal of Endocrinology (2008) 198, 83-89

\section{Introduction}

Regulation of prolactin secretion from the anterior pituitary in lactating females is not fully recognized. It is accepted that the hypothalamic neuroendocrine dopaminergic (NEDA) system exerts a dominant and inhibitory effect on prolactin and that prolactin acts in a short-loop feedback manner to regulate its own secretion by stimulating the NEDA system (Freeman et al. 2000). Studies on rodents showed that during late pregnancy, the NEDA system had a reduced ability to respond to prolactin (Grattan \& Averill 1995, Andrews et al. 2001) and that this impaired negative feedback continued into lactation (Arbogast \& Voogt 1996, Anderson et al. 2006), allowing the maintenance of increased prolactin secretion to promote lactogenesis. Increased secretion of prolactin is also responsible for the stimulation of maternal behaviour and may contribute to the suppression of the stress response during lactation (Grattan 2002). Maintenance of increased lactotroph activity during lactation seems to require additional factor(s) enhancing prolactin synthesis and release. Recently, some hypothalamic compounds are thought to play the role of prolactin-releasing factor (PRF; Maruyama et al. 1999, Curlewis et al. 2002, Samson et al. 2003) and amongst them, salsolinol that paradoxically may be produced by the inhibitory NEDA system (Mravec 2006).

As reported by Naoi et al. (1996, 2002), salsolinol can be synthesized from dopamine (DA) and acetaldehyde or alternatively, from DA and pyruvic acid by forming an intermediate metabolite, salsolinol-1-carboxylic acid. This DA-originated compound, which is well known from its involvement in the progression of a disease characterized by dysfunctional dopaminergic neurons (Moser et al. 1995, Antkiewicz-Michaluk 2002), was found to be present in neuro-intermediate lobe (NIL) as well as median eminence (ME) extracts of male, intact and ovariectomized female rats (Toth et al. 2001). Salsolinol concentration has been shown to be elevated during situations when prolactin secretion is increased from the anterior lobe of the pituitary gland (Toth et al. 2001), and salsolinol binds specifically with the pituitary cells of lactating rats (Toth et al. 2002, Homicsko et al. 2003). 
More recently, it has been shown that a cAMP-coupled mechanism is probably involved in the prolactin-releasing action of salsolinol at the level of lactotrophs (Randai et al. 2005). In the current study, we hypothesized that salsolinol, as a putative neurotransmitter, was present in the infundibular nucleus IN/ME of lactating ewes and that its extracellular concentration in the IN/ME could increase in response to suckling stimulus in a manner similar to plasma concentration of prolactin. In addition, the secretion of growth hormone $(\mathrm{GH})$ was monitored throughout the experiment since both prolactin and $\mathrm{GH}$ are known to respond to suckling (Wehrenberg \& Gaillard 1989, Negrao \& Marnet 2002).

\section{Materials and Methods}

\section{Animals and managements}

All animal procedures were conducted in accordance with the Polish Guide for the Care and Use of Animals (1997) and approved by the Local Ethics Committee.

Seven mature ewes (3- to 4-year-old) were mated naturally in September and lambed in the following February. They were maintained indoors in individual pens under natural lighting conditions $\left(52^{\circ} \mathrm{N}, 21^{\circ} \mathrm{E}\right)$. The animals were fed twice daily a diet formulated to provide $100 \%$ of the National Research Institute of Animal Production recommendations for pregnancy and lactation (Norms 1993) with water available ad libitum. All ewes were implanted bilaterally (left and right) with a stainless steel guide canullae $(1.4 \mathrm{~mm}$ o.d.) into the IN/ME during the second month of pregnancy. The implantation was performed under general anaesthesia, through a drill hole in the skull, in accordance with the stereotaxic co-ordinate system for sheep hypothalamus (Welento et al. 1969) and procedure described by Traczyk \& Przekop (1963), positions: frontal, 29.5-31 $\mathrm{mm}$ and sagittal, $1.2 \mathrm{~mm}$. The guide canullae were fixed to the skull with stainless steel screws and dental cement. The external opening to the canal was closed with a stainless steel cap. The placement of the push-pull cannula was confirmed after slaughtering (after weaning), by the infusion of a small volume of blue ink.

\section{Experimental design}

Perfusions of the IN/ME were made with the Ringer-Locke solution by the push-pull method (Misztal et al. 2004). All ewes were perfused twice (two sides) with a 3-day interval between 28 and 32 days of lactation. The tubes for perfusates contained $50 \mu \mathrm{l}$ of $0 \cdot 1 \mathrm{mM}$ ascorbic acid, an antioxidant for catecholamines and were kept in an ice bath during sampling. The flow rate was $7 \mu \mathrm{l} / \mathrm{min}$ and the volume of one perfusate collected during the $30-\mathrm{min}$ period was about $250 \mu \mathrm{l}$. The total time of perfusion was $6 \mathrm{~h}$, including a pre-perfusion period from 0900 to $1000 \mathrm{~h}$ to eliminate the changes in catecholamines release caused by insertion of the push-pull cannula and the collecting period from 1000 to $1500 \mathrm{~h}$. The collecting period in every ewe consisted of control non-suckling period, from 1000 to $1230 \mathrm{~h}$ (five perfusates), and suckling period, from 1230 to $1500 \mathrm{~h}$ (next five perfusates). Immediately after filling, the tubes were frozen in liquid nitrogen and stored at $-80{ }^{\circ} \mathrm{C}$ until assayed for salsolinol and DA.

During the experiment, nursing sheep were kept together with their lamb(s) in comfortable cages, where they could lie down and had unrestrained access to hay. Lamb(s) had restrained access to mother's udder from 0900 to $1230 \mathrm{~h}$, having a visual and tactile contact in front of mother. After this time, $\operatorname{lamb}(\mathrm{s})$ were allowed to suck mother's milk (always from the sixth perfusate). The suckling was then monitored to the end of the experiment. Simultaneously, blood samples were collected from 1000 to $1500 \mathrm{~h}$ at $10-$ min intervals, through a catheter inserted into the jugular vein a day before the experiment. After centrifugation in heparinized tubes, plasma was stored at $-20^{\circ} \mathrm{C}$ until prolactin and $\mathrm{GH}$ assay. Additionally, in four selected ewes, perfusion was performed 10 weeks after weaning. All perfusions were done with calibrated $1.0 \mathrm{ml}$ gas-tight syringes and a CMA/100 microinjection pump (Stockholm, Sweden).

Post-mortem localization of the push-pull cannula revealed that in four ewes the perfusion site was located just behind the optic chiasm, in the anterior part of the IN/ME and in three ewes in the posterior part of the IN/ME (Fig. 1). The tip of the push-pull cannula inserted on the day of perfusion was placed within the IN on each side of the brain. Because an area of about $1.5 \mathrm{~mm}$ in diameter - IN and some part of ME proximate to IN - could be perfused, these formations were considered as the site of perfusion. Four series of perfusates $(n=4)$ collected from the anterior part of the IN/ME (unilateral collection from four ewes) and

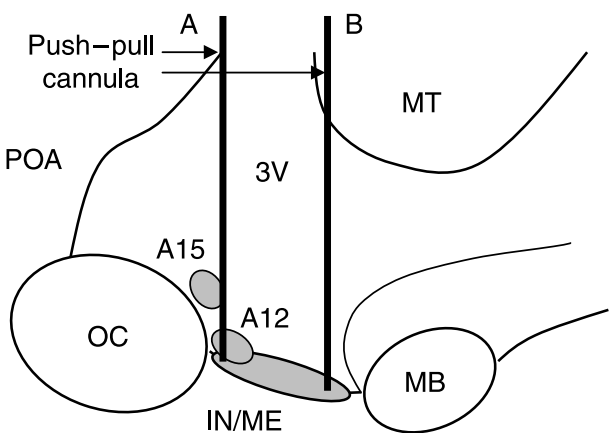

Figure 1 Diagram of the sheep hypothalamus showing a placement of the push-pull cannula: the anterior (A) and posterior (B) parts of the infundibular nucleus-median eminence (IN/ME). A12 and A15, dopaminergic nuclei; MB, mammillary body; MT, medial thalamus; OC, optic chiasm; POA, preoptic area; $3 \mathrm{~V}$, third cerebral ventricle. 
six series of perfusates $(n=6)$ collected from the posterior part (bilateral collection from three ewes) were taken to be analyzed.

\section{Analytical techniques}

The concentrations of salsolinol as well as DA in the perfusates were analyzed using high performance liquid chromatography (HPLC) with electrochemical detection (Tomaszewska-Zaremba et al. 2002). Perfusates were centrifuged for $15 \mathrm{~min}$ and filtered through a $0 \cdot 22 \mu \mathrm{m}$ membrane GVWP; $50 \mu \mathrm{l}$ aliquots of each filtrate were injected into a LC-18-DB $(15 \mathrm{~cm} \times 4.6 \mathrm{~mm}$ ID, $5 \mu \mathrm{m})$ Supelco column protected by a superguard LC-18-DB $5 \mu \mathrm{m}$ Supercosil, $2 \mathrm{~cm}$ precolumn. The column was coupled with an electrochemical detector (Hewlett Packard HP 1049 A Programmable Electrochemical Detector, Santa Clara, CA, USA) equipped with a glassy carbon working electrode and $\mathrm{Ag} / \mathrm{AgCl}$ reference electrode. The oxidative potential of the electrochemical detector was $0 \cdot 650 \mathrm{~V}$. Samples were eluted isocratically with a mobile phase consisting of $0.01 \mathrm{~mol} / 1 \mathrm{NaCl}, 0.001 \mathrm{~mol} / 1 \mathrm{EDTA}$, $53-55 \mathrm{mg} / 1$ octal sulphate sodium salt, $12 \% \mathrm{CH}_{3} \mathrm{OH}$; the $\mathrm{pH}$ of the mobile phase was $3 \cdot 6$. The mobile phase was filtered through a $0.22 \mu \mathrm{m}$ GVWP membrane and degassed under vacuum with ultrasonic agitation. The flow rate was $0.8 \mathrm{ml} / \mathrm{min}$. Stock solutions of standards were stored at $-20{ }^{\circ} \mathrm{C}$. The limits of detection were $10 \mathrm{pg} / 50 \mu \mathrm{l}$ for salsolinol and $5 \mathrm{pg} / 50 \mu \mathrm{l}$ for DA.

The concentration of prolactin in plasma was assayed by the RIA double-antibody method, using anti-ovine prolactin and antirabbit $\gamma$-globulin antisera as described by Wolińska et al. (1977). The prolactin standard was synthesized and kindly provided by Prof Kazimierz Kochman from our Institute (Kochman \& Kochman 1977). The assay sensitivity for prolactin was $2 \mathrm{ng} / \mathrm{ml}$ and the intra- and inter-assay coefficients of variation were 9 and $12 \%$ respectively.

The concentration of GH in plasma was assayed by the RIA double-antibody method, using anti-bovine GH and antirabbit $\gamma$-globulin antisera and bovine $\mathrm{GH}$ standard (NIDDK-GH-B-1003A). The full characteristics of the antiserum and method were described by Dvorak et al. (1978). The assay sensitivity for $\mathrm{GH}$ was $0 \cdot 6 \mathrm{ng} / \mathrm{ml}$, and the intra- and inter-assay coefficients of variation were 5.9 and $10 \cdot 2 \%$ respectively.

\section{Statistical analysis}

Because of the different localization of dopaminergic nuclei in the ovine hypothalamus (Thiery 1991), the statistical analysis for salsolinol was done separately for the anterior $(n=4)$ and posterior $(n=6)$ parts of the IN/ME, while the changes in the concentrations of prolactin and GH were analyzed together $(n=10)$ due to the same protocol of the experiment used for all ewes. The effect of suckling on salsolinol concentration was first examined by ANOVA for repeated measurements and then for consecutive perfusates by one-factor ANOVA followed by the Tukey's post hoc test (Statistica, StatSoft, Inc., Tulsa, OK, USA). The effect of suckling on the secretion of prolactin and $\mathrm{GH}$ was examined by ANOVA for repeated measurements. The Tukey's post hoc test was used for comparisons of plasma prolactin and GH concentrations between the 30-min periods, corresponding to the consecutive perfusates. All data are expressed as means \pm s.E.M.

\section{Results}

\section{Suckling}

Episodic suckling was observed during the second part of perfusion, from 1230 to $1500 \mathrm{~h}$, when lamb(s) were allowed to suck mother's milk. However, the most intensive suckling occurred at the beginning (first $30 \mathrm{~min}$ ) and during the last hour of this period.

\section{Perfusate salsolinol and $\mathrm{D} A$ concentrations}

The means $( \pm$ s.E.M.) concentration of salsolinol in perfusates collected from the anterior part of the IN/ME $(n=4)$ during the non-suckling period was $140 \cdot 92 \pm 26 \cdot 67 \mathrm{pg} / 50 \mu \mathrm{l}$ perfusate and increased significantly $(P<0 \cdot 05)$ during the suckling period to $293 \cdot 42 \pm 47 \cdot 84 \mathrm{pg} / 50 \mu \mathrm{l}$ perfusates. Due to high fluctuations in the release of salsolinol in the individual animals, there were no significant differences in the mean concentrations of this compound between the consecutive perfusates. However, a clear tendency for an increase in the salsolinol concentration was visible, at least during the first hour of suckling (sixth and seventh perfusate, Fig. 2).

The concentration of salsolinol in perfusates collected from the posterior part of the IN/ME $(n=6)$ during the nonsuckling period was $13 \cdot 32 \pm 2.90 \mathrm{pg} / 50 \mu \mathrm{l}$ perfusate and increased significantly $(P<0 \cdot 001)$ during the suckling period to $96 \cdot 70 \pm 8 \cdot 52 \mathrm{pg} / 50 \mu \mathrm{l}$ perfusates. The mean concentrations of salsolinol in consecutive perfusates collected from the posterior part during suckling were significantly $(P<0 \cdot 05$, $P<0.01$ and $P<0 \cdot 001$ ) higher than the concentrations noted in perfusates collected before suckling (Fig. 2). Interestingly, the extracellular content of salsolinol in the posterior part of the IN/ ME was lower than that in the anterior part. The mean patterns of salsolinol release detected in both parts of the IN/ME reflect the different salsolinol responses to suckling stimulus in individual ewes, as shown in Fig. 3.

While DA was not detected in perfusates collected from the anterior part of the IN/ME, only vestigial amounts of DA were found in a few individual perfusates collected from the posterior part of the IN/ME during the non-suckling period (data not shown). On the contrary, salsolinol was not detected in the perfusates collected from the IN/ME of ewes $(n=4) 10$ weeks after weaning (seasonal anoestrus), while the mean DA concentration in particular ewes ranged from $35 \cdot 66 \pm 7 \cdot 51$ to $90 \cdot 0 \pm 36 \cdot 40 \mathrm{pg} / 50 \mu \mathrm{l}$ of perfusate. 

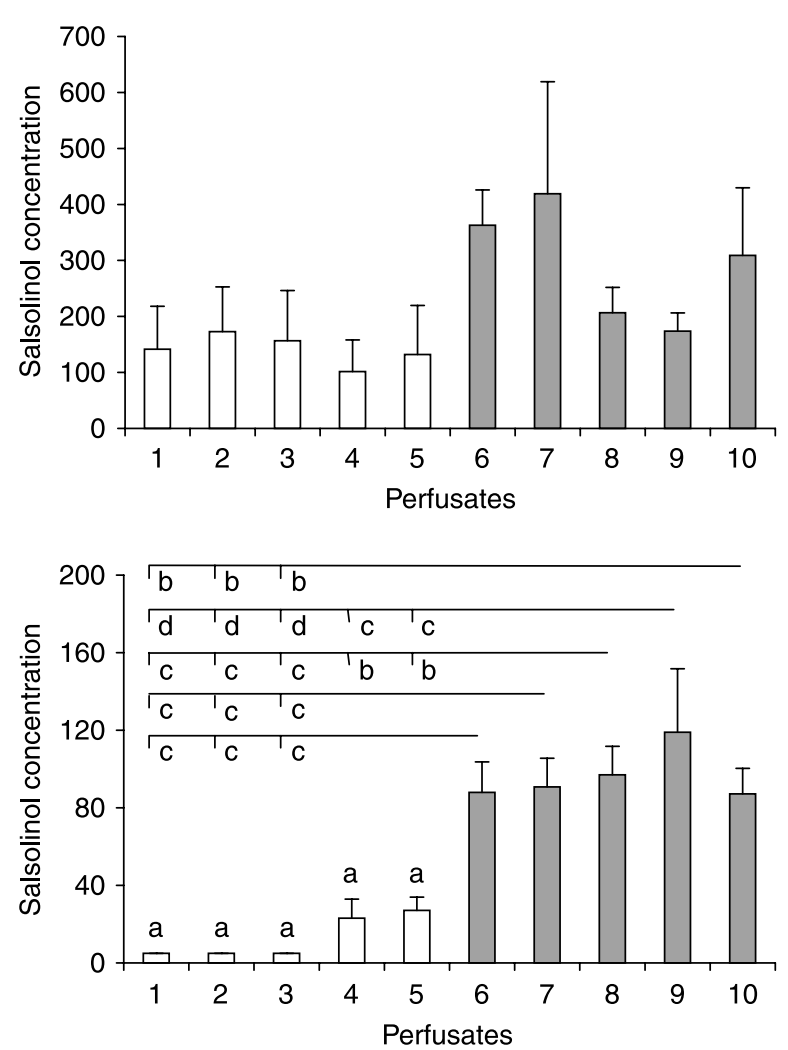

Figure 2 Mean salsolinol concentrations $(\mathrm{pg} / 50 \mu \mathrm{l})$ in perfusates collected from the anterior ( $n=4$, top) and posterior ( $n=6$, bottom) parts of the infundibular nucleus-median eminence (IN/ME) of lactating ewes during the non-suckling (1000-1230 h, white bars) and suckling (1230-1500 h, grey bars) periods. Note that salsolinol concentration in the anterior part is higher than in the posterior part of the IN/ME. ab, $P<0 \cdot 05 ; \mathrm{ac}, P<0 \cdot 01$; ad, $P<0 \cdot 001$.

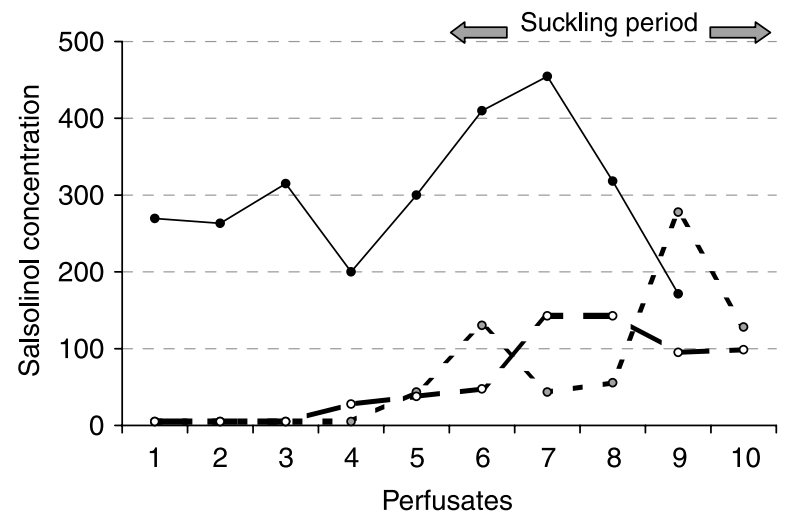

Figure 3 Representative patterns of salsolinol release within the infundibular nucleus-median eminence (IN/ME) in individual lactating ewes during the non-suckling (1000-1230 h) and suckling (1230-1500 h, grey arrows) periods: one represents an anterior part (black circles) and two other represent a posterior part (white and grey circles) of the IN/ME.
Plasma prolactin and GH concentrations (all ewes)

The mean plasma prolactin concentration during the nonsuckling period was $93.35 \pm 2.91 \mathrm{ng} / \mathrm{ml}$ and increased significantly $(P<0 \cdot 001)$ during the suckling period to $146 \cdot 10 \pm 5 \cdot 84 \mathrm{ng} / \mathrm{ml}$. Prolactin concentration increased significantly $(P<0 \cdot 01$ and $P<0 \cdot 001)$ during the first $10-30 \mathrm{~min}$ of suckling (corresponding to the sixth perfusate) and maintained on a high level to the end of the experiment, when compared with the concentrations noted during the consecutive 30-min periods before suckling (Fig. 4).

The mean plasma GH concentration before suckling was $4 \cdot 42 \pm 0 \cdot 25 \mathrm{ng} / \mathrm{ml}$ and increased significantly $(P<0 \cdot 001)$ during the suckling period to $7.58 \pm 0.46 \mathrm{ng} / \mathrm{ml}$. As in the case of prolactin, GH concentration increased significantly $(P<0 \cdot 001)$ during the first $10-30 \mathrm{~min}$ of suckling period, when compared with the concentrations noted during the consecutive 30-min periods before suckling. Interestingly, marked increases in $\mathrm{GH}$ concentration corresponded to two periods of intensive suckling $(P<0 \cdot 01$ and $P<0 \cdot 001$, Fig. 4).
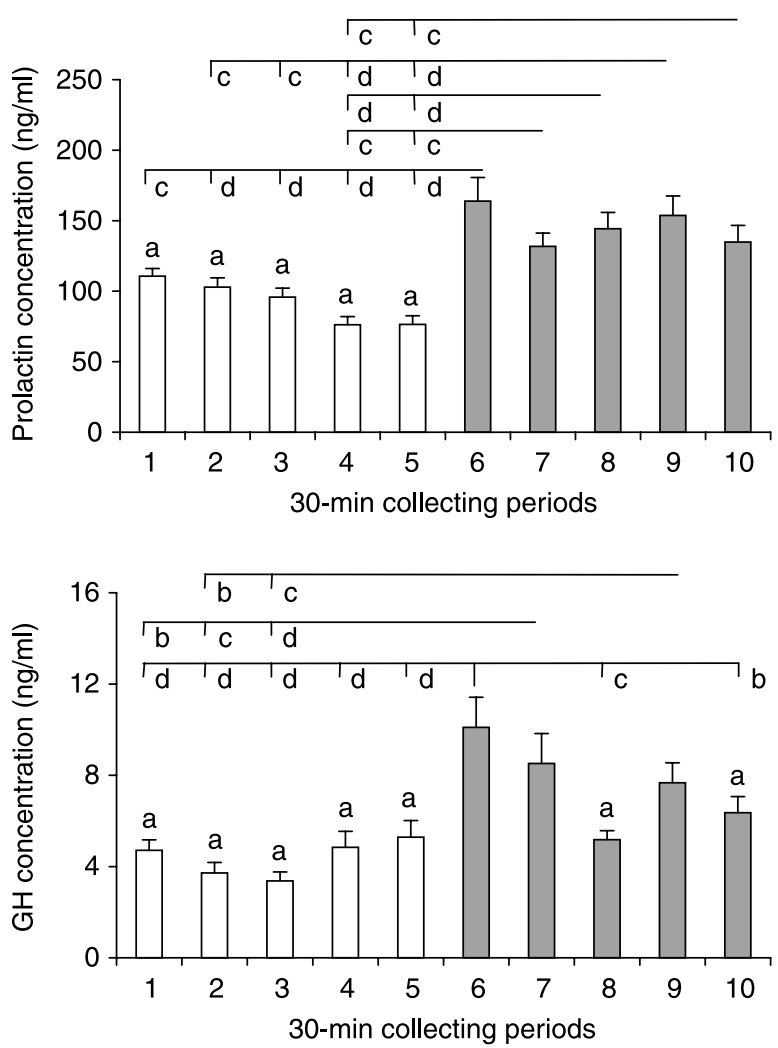

Figure 4 Mean plasma concentrations of prolactin (top) and $\mathrm{GH}$ (bottom) in lactating ewes $(n=10)$ during the consecutive 30-min periods from 1000 to $1500 \mathrm{~h}$ : non-suckling period 1000-1230 h (white bars); suckling period 1230-1500 h (grey bars). Blood samples collected every $10 \mathrm{~min}$. ab, $P<0.05$; ac, $P<0 \cdot 01$; ad, $P<0 \cdot 001$. 

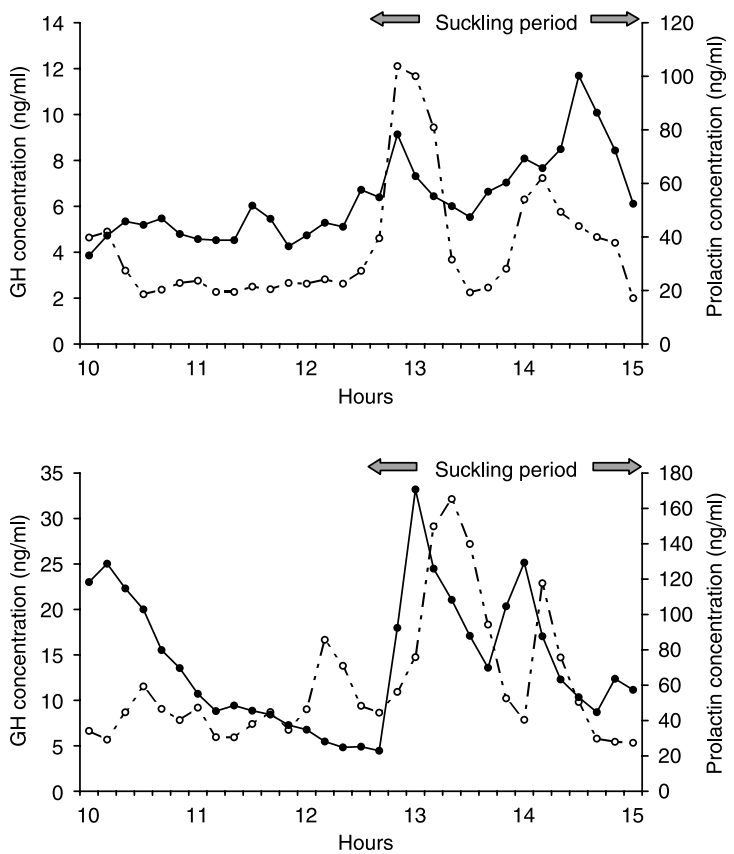

Figure 5 Representative patterns of prolactin (black circles) and $\mathrm{GH}$ (white circles) secretion in individual lactating ewes during the nonsuckling (1000-1230 h) and suckling (1230-1500 h, grey arrows) periods. Note hormonal concentrations in ewe nursing twins (bottom) are higher than in ewe nursing a single lamb (top).

Representative individual patterns of prolactin and $\mathrm{GH}$ secretion in ewes during non-suckling and suckling periods are shown in Fig. 5. In most of the cases, there was a biphasic increase in prolactin and GH concentrations which corresponded to periods of intensive suckling (see above).

\section{Discussion}

This study provides the first data on the presence of salsolinol, a derivative of DA, in the hypothalamus of lactating ewes. As hypothesized, an increase in the extracellular concentration of this compound within the IN/ME occurred in response to suckling, and it was closely related to the changes in plasma concentration of prolactin and in part to these in $\mathrm{GH}$ concentration.

The role of prolactin and GH in maintenance of lactation in sheep is well established. In this species, both these hormones seem to be important for galactopoiesis process (Hooley et al. 1978, Tucker 1994). Suckling of the nipple by the neonate or milking is one of the most important factors, providing a powerful prolactin stimulus, involved in maintaining a prolonged state of hyperprolactinaemia (Grattan 2002). Investigating a temporal course of prolactin and $\mathrm{GH}$ secretion in the individual ewes, our data show a high similarity to those obtained on lactating rats (Saunders et al. 1976, Riskind et al. 1984, Wehrenberg \& Gaillard 1989) or cows (Negrao \&
Marnet 2002). It has been proposed that suckling or milking works by decreasing prolactin-inhibiting factor (DA) from the hypothalamus, and thereby increasing prolactin levels. They may also act to increase the effectiveness of any putative PRF.

For years, the attention has been focused on the activity of the NEDA system in lactating females. Classically, the NEDA system secretes DA, a dominant suppressor of the synthesis and release of prolactin. An activity of the NEDA is under feedback control by prolactin, creating together a regulatory feedback mechanism (Freeman et al. 2000). Prolactin through binding to its receptors specifically activates signal transducer and activator of transcription 5b (STAT5b) in dopaminergic neurons, leading to the increased expression and activity of tyrosine hydroxylase, the rate-limiting enzyme in DA synthesis (Ma et al. 2005a,b). During lactation, dopaminergic neurons are unresponsive to prolactin (Arbogast \& Voogt 1996), which results from reduced STAT5b signalling due to increased expression of cytokine-inducible $\mathrm{SH} 2$ domaincontaining protein (CIS; Anderson et al. 2006). This last molecule is a member of the family of proteins known to negatively regulate prolactin signalling. This study demonstrated that in lactating rats, CIS level in dopaminergic, neurons increased in the presence of suckling pups (Anderson et al. 2006).

There is a growing number of experimental data suggesting that the dopaminergic regulatory process of prolactin secretion during lactation may be mediated by salsolinol. This catechol isoquinoline may be synthesized directly from DA and acetaldehyde or indirectly, forming salsolinol-1-carboxylic acid and further to salsolinol (Naoi et al. 1996, 2002). Although not all enzymes of an indirect pathway of salsolinol synthesis are known, this compound and its metabolites can be detected in many areas of the brain that are also rich in DA (Toth et al. 2001, Naoi et al. 2002). In the current study, we have shown that salsolinol is present in the IN/ME of lactating ewes. A higher concentration of salsolinol and its variability found in the anterior than the posterior part of the IN/ME suggest that the main source of this compound may be the dopaminergic cells from the IN A12 and/or these from the retrochiasmatic area (Thiery 1991). Our data did not show, however, a direct proof for salsolinol synthesis in these dopaminergic nuclei. So far, the presence of salsolinol in the ME has been shown in rats (Toth et al. 2001). Musshoff et al. (2000), studying human brain areas involved in the aetiology of alcoholism and Parkinsonism have also found salsolinol present in the hypothalamus. Also, NIL of the pituitary gland in rats (Toth et al. 2001) and more recently the posterior pituitary in bovine (Hashizume et al. 2008) have been indicated as the sites of salsolinol detection. However, salsolinol found in bovine pituitary extract seemed to originate from the hypothalamic dopaminergic neuron terminals rather than be synthesized in the gland. The presence of salsolinol in the IN/ME of our ewes does not preclude that it can be detected within the ovine posterior pituitary, although efferent projections of the NEDA system have also been found in this structure (Gayrard et al. 1995). Interestingly, in our lactating ewes, no DA or only slight amounts of DA were detected in 
perfusates collected from the IN/ME. On the contrary, a lack of extracellular salsolinol and a presence of DA were demonstrated 10 weeks after weaning. It indicates how important and significant changes in the enzymatic pathways occur within the NEDA system between the non-lactating and lactating periods and vice versa.

Data from our in vivo experiment showed that extracellular concentration of salsolinol in the IN/ME increased parallel to plasma prolactin and $\mathrm{GH}$ concentrations during the period of suckling. The distinct peaks observed in the patterns of salsolinol release in individual ewes corresponded to an intensive suckling stimulus. Although there was no relationship between the number of lambs nursed and salsolinol level, the prolactin response in ewes nursing twins was more potent than in ewes nursing a single lamb. Toth et al. (2001) found that salsolinol concentrations in NIL of lactating rats revealed parallel increases with plasma prolactin in response to a brief suckling stimulus following $4-\mathrm{h}$ separation. They also demonstrated that salsolinol can elevate prolactin release in pituitary cell cultures as well as in hypophysectomized rats bearing anterior lobe transplants under the kidney capsule. Moreover, $\left[{ }^{3} \mathrm{H}\right]$-salsolinol has been shown to bind specifically to the homogenate of the anterior lobe as well as NIL obtained from lactating rats (Toth et al. 2001, 2002, Homicsko et al. 2003). Recently, Hashizume et al. (2008) demonstrated that a single i.v. injection of salsolinol significantly and dose-dependently stimulated the release of prolactin in goats, and the intracerebroventricular treatment stimulated prolactin release in calves. Taken together, ours and other data show that in mammals, in specific physiological conditions, salsolinol may play the role of PRF. Especially, during lactation, salsolinol may mediate suckling-induced release of prolactin.

To our knowledge, there is no data linking hypothalamic salsolinol release with regulation of $\mathrm{GH}$ secretion. According to data of Toth et al. (2001) obtained in rats, salsolinol did not affect the secretion of other pituitary hormones, except prolactin. Our results only focus attention on some details in the pattern of $\mathrm{GH}$ response to suckling, which may relate to salsolinol presence within the hypothalamus. Similarly to prolactin, the secretion of $\mathrm{GH}$ increased during the periods of intensive suckling and what was interesting, $\mathrm{GH}$ rise was in some cases abrupt and more potent than that of prolactin. Participation of salsolinol in the regulatory process of $\mathrm{GH}$ release in lactating ewes, if possible, could affect its action on the neurons synthesizing GH-releasing hormone and/or somatostatin. The evidence exists that catecholamines are involved in the modulation of the release of these two neuropeptides (McMahon et al. 2001). It requires, however, further detailed research on the salsolinol action in ovine hypothalamus in different physiological stages.

In conclusion, our current study reveals that DA-derived salsolinol is present in the IN/ME of lactating ewes and that its extracellular concentration increases during suckling. Moreover, it supports the role of salsolinol as a neurotransmitter involved in the regulatory process of prolactin secretion at least during lactation.

\section{Declaration of Interest}

The authors declare that there is no conflict of interest that would prejudice the impartiality of this scientific work.

\section{Funding}

This work was supported by Grant No.2 PO6D 02930 (Ministry of Science and Higher Education, Poland).

\section{Author contribution statement}

All co-authors declare that part of the presented results was obtained during the $\mathrm{PhD}$ course of $\mathrm{K} \mathrm{G}$ and express the agreement on location them in his $\mathrm{PhD}$ thesis.

\section{Acknowledgements}

The authors would like to express their thanks to veterinary surgeon J Rutkowski for help in the brain surgery.

\section{References}

Anderson ST, Barclay JL, Fanning KJ, Kusters DHL, Waters MJ \& Curlewis JD 2006 Mechanisms underlying the diminished sensitivity to prolactin negative feedback during lactation: reduced STST5 signalling and up-regulation of cytokine-inducible SH2 domain-containing protein (CIS) expression in tuberoinfundibular dopaminergic neurons. Endocrinology 147 1195-1202.

Andrews ZB, Kokay IC \& Grattan DR 2001 Dissociation of prolactin secretion from tuberoinfundibular dopamine activity in late pregnant rats. Endocrinology 142 2719-2724.

Antkiewicz-Michaluk L 2002 Endogenous risk factors in Parkinson's disease: dopamine and tetrahydroisoquinolines. Polish Journal of Pharmacology $\mathbf{5 4}$ 567-572.

Arbogast LA \& Voogt JL 1996 The responsiveness of tuberoinfundibular dopaminergic neurons to prolactin feedback is diminished between early lactation and midlactation in the rat. Endocrinology 137 47-54.

Curlewis JD, Kusters DHL, Barclay JL \& Anderson ST 2002 Prolactinreleasing peptide in the ewe: cDNA cloning, mRNA distribution and effects on prolactin secretion in vitro and in vivo. Journal of Endocrinology 174 45-53.

Dvorak P, Becka S, Krejci P \& Chrpova M 1978 Radioimmunoassay of bovine growth hormone. Radiochemical and Radioanalytical Letters 34 155-160.

Freeman ME, Kanyicska B, Lenart A \& Nagy G 2000 Prolactin structure function and regulation of secretion. Physiological Reviews 80 1523-1631.

Gayrard V, Thiery J-C, Thibault J \& Tillet Y 1995 Efferent projections from the retrochiasmatic area to the median eminence and to the pars nervosa of the hypophysis with special reference to the A15 dopaminergic cell group in the sheep. Cell and Tissue Research 281 561-567.

Grattan DR 2002 Behavioural significance of prolactin signalling in the central nervous system during pregnancy and lactation. Reproduction 123 497-506

Grattan DR \& Averill RL 1995 Absence of short-loop autoregulation of prolactin during late pregnancy in the rat. Brain Research Bulletin 36 413-416

Hashizume T, Shida R, Suzuki S, Nokana S, Yonezawa C, Yamashita T, Kasuya E, Sutoh M, Olah M, Szekacs D et al. 2008 Salsolinol is present in the bovine posterior pituitary gland and stimulates the release of prolactin both in vivo and in vitro in ruminants. Domestic Animal Endocrinology 34 146-152. 
Homicsko KG, Kertesz I, Randai B, Toth BE, Toth G, Fulop F, Fekete MI \& Nagy GM 2003 Binding site of salsolinol: its properties in different regions of the brain and the pituitary gland of the rat. Neurochemistry International $\mathbf{4 2}$ $19-26$.

Hooley RD, Campbell JJ \& Findlay JK 1978 The importance of prolactin for lactation in the ewe. Journal of Endocrinology 79 301-310.

Kochman H \& Kochman K 1977 Purifications of ovine and bovine prolactins on DEAE cellulose chromatography and preparative polyacrylamide gel electrophoresis. Bulletin de l'Acad?mie Polonaise des Sciences. S?rie des Sciences Biologiques 25 67-70.

Ma FY, Grattan DR, Goffin V \& Bunn SJ 2005a Prolactin-regulated tyrosine hydroxylase activity and messenger ribonucleic acid expression in mediobasal hypothalamic cultures: the differential role of specific protein kinases. Endocrinology 146 93-102.

Ma FY, Anderson GM, Gunn TD, Goffin V, Grattan DR \& Bunn SJ $2005 b$ Prolactin specifically activates signal transducer and activator of transcription $5 \mathrm{~b}$ in neuroendocrine dopaminergic neurons. Endocrinology 146 5112-5119.

Maruyama M, Matsumoto H, Fujiwara K, Kitada C, Hinuma S, Onda H, Fujino M \& Inoue K 1999 Immunocytochemical localization of prolactinreleasing peptide in the rat brain. Endocrinology 140 2326-2333.

McMahon CD, Radcliff RP, Lookingland KJ \& Tucker HA 2001 Neuroregulation of growth hormone secretion in domestic animals. Domestic Animal Endocrinology 20 65-87.

Misztal T, Romanowicz K, Tomaszewska-Zaremba D, Wojcik-Gladysz A \& Barcikowski B 2004 The effects of prolonged, intracerebroventricular prolactin treatment on luteinizing hormone secretion, catecholaminergic activity and estrous behavior in ewes. Experimental and Clinical Endocrinology and Diabetes 112 215-221.

Moser A, Scolz J, Nobbe F, Vieregge P, Bohme V \& Bamberg H 1995 Presence of $N$-methyl-norsalsolinol in the CSF: correlations with dopamine metabolites of patients with Parkinson's disease. Journal of the Neurological Sciences 131 183-189.

Mravec B 2006 Salsolinol, a derivate of dopamine, is a possible modulator of catecholaminergic transmission: a review of recent developments. Physiological Research 55 353-364.

Musshoff F, Schmidt P, Dettmeyer R, Priemer F, Jachau K \& Madea B 2000 Determination of dopamine and dopamine-derived (R)-/(S)-salsolinol and norsalsolinol in various human brain areas using solid-phase extraction and gas chromatography/mass spectrometry. Forensic Science International $\mathbf{1 1 3}$ 359-366.

Naoi M, Maruyama W, Dostert P, Kohda K \& Kaiya T 1996 A novel enzyme enantio-selectively synthesizes ${ }^{\circledR}$ salsolinol, a precursor of a dopaminergic neurotoxin, N-methyl-(R)salsolinol. Neuroscience Letters 212 183-186.

Naoi M, Maruyama W, Akao Y \& Yi H 2002 Dopamine-derived endogenous $\mathrm{N}$-methyl-R salsolinol: its role in Parkinson's disease. Neurotoxicology and Teratology 24 579-591.

Negrao JA \& Marnet PG 2002 Effect of calf suckling on oxytocin, prolactin, growth hormone and milk yield in crossbred Gir x Holstein cows during milking. Reproduction, Nutrition, Development 42 373-380.
Randai B, Kandar Z, Somogyvari-Vigh A, Mergl Z, Olah M, Fulop F, Vecsernyes M \& Nagy GM 2005 Salsolinol induces a decrease in cyclic AMP at the median eminence and an increase at the adenohypophysis in lactating rats. Brain Research Bulletin 65 105-110.

Riskind PN, Millard WJ \& Martin JB 1984 Opiate modulation of the anterior pituitary hormone response during suckling in the rat. Endocrinology 114 1232-1237.

Samson WK, Taylor MM \& Baker JR 2003 Prolactin-releasing peptides. Regulatory Peptides 114 1-5.

Saunders A, Terry LC, Audet J, Brazeau P \& Martin JB 1976 Dynamic studies of growth hormone and prolactin secretion in the female rat. Neuroendocrinology 21 193-196.

Thiery J-C 1991 Monoamine content of the stalk-median eminence and hypothalamus in adult female sheep as affected by daylength. Journal of Neuroendocrinology 3 407-411.

Tomaszewska-Zaremba D, Mateusiak K \& Przekop F 2002 The involvement of GABAA receptors in the control of $\mathrm{GnRH}$ and $\beta$-endorphin release, and catecholaminergic activity in the preoptic area in anestrous ewes. Experimental and Clinical Endocrinology and Diabetes 110 336-342.

Toth BE, Homicsko K, Randai B, Maruyama W, DeMaria JE, Vecsernyes M, Fekete MI, Fulop F, Naoi M, Freeman ME et al. 2001 Salsolinol is a putative endogenous neuro-intermediate lobe prolactin-releasing factor. Journal of Neuroendocrinology 13 1042-1050.

Toth BE, Bodnar I, Homicsko K, Fulop F, Fekete MIK \& Nagy GM 2002 Physiological role of salsolinol: its hypophysiotrophic function in the regulation of pituitary prolactin secretion. Neurotoxicology and Teratology $\mathbf{2 4}$ 655-666.

Traczyk W \& Przekop F 1963 Methods of investigation of the function of the hypothalamus and hypophysis in chronic experiments in sheep. Acta Physiologica Polonica 14 217-226.

Tucker HA 1994 Lactation and its hormonal control. In The Physiology of Reproduction, vol 2, edn 2, pp 1065-1098. Eds Knobil E \& Neill JD. New York: Raven Press.

Wehrenberg WB \& Gaillard RC 1989 Neuroendocrine mechanisms regulating growth hormone and prolactin secretion during lactation. Endocrinology 124 464-467.

Welento J, Szteyn S \& Milart Z 1969 Observations on the stereotaxic configuration of the hypothalamus nuclei in the sheep. Anatomischer Anzeiger 124 1-27.

Wolińska E, Polkowska J \& Domański E 1977 The hypothalamic centers involved in the control of production and release of prolactin in sheep. Journal of Endocrinology 73 21-29.

Received in final form 25 March 2008

Accepted 22 April 2008

Made available online as an Accepted Preprint 23 April 2008 